\title{
Intratracheal Transplantation of Alveolar Type II Cells Reverses Bleomycin-induced Lung Fibrosis
}

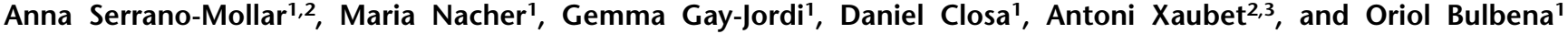 \\ ${ }^{1}$ Department of Experimental Pathology, Institut d'Investigacions Biomèdiques de Barcelona, Consejo Superior de Investigaciones Científicas, \\ Institut d'Investigacions Biomèdiques August Pi i Sunyer, Barcelona, Spain; ${ }^{2}$ Centro de Investigación Biomédica en Red (CIBER) \\ de Enfermedades Respiratorias, Barcelona, Spain; and ${ }^{3}$ Servei de Pneumologia, Institut Clínic del Tórax, Hospital Clínic, Barcelona, Spain
}

\begin{abstract}
Rationale: Transplantation of stem cells has been proposed as a strategy for repair of lung fibrosis. Nevertheless, many studies have yielded controversial results that currently limit the potential use of these cells as an efficient treatment. Alveolar type II cells are the progenitor cells of the pulmonary epithelium and usually proliferate after epithelial cell injury. During lung fibrosis, however, the altered regeneration process leads to uncontrolled fibroblast proliferation. Objectives: To investigate whether intratracheal transplantation of isolated alveolar type II cells can halt and reverse the fibrotic process in an experimental model of bleomycin-induced lung fibrosis in rats. Methods: Lung fibrosis was induced in syngeneic female Lewis rats by a single intratracheal instillation of bleomycin $(2.5 \mathrm{U} / \mathrm{kg})$. Animals were transplanted with alveolar type II cells from male animals at a dose of $2.5 \times 10^{6}$ cells per animal 3, 7, and 15 days after endotracheal bleomycin instillation. Animals were killed 21 days after the induction of lung fibrosis.

Measurements and Main Results: Lung fibrosis was assessed by histologic study and determination of hydroxyproline content. Engraftment of transplanted cells was measured by real-time polymerase chain reaction for the $Y$ chromosome and by fluorescence in situ hybridization for the $\mathrm{Y}$ chromosome. Transplantation of alveolar type II cells into damaged lung 3, 7, or 15 days after bleomycin instillation led to reduced collagen deposition, and reduction in the severity of pulmonary fibrosis.

Conclusions: This study demonstrates the potential role of alveolar type II cell transplantation in designing future therapies for lung fibrosis.
\end{abstract}

Keywords: cell therapy; lung epithelium; lung repair

Idiopathic pulmonary fibrosis is a chronic inflammatory interstitial lung disease that has a potentially fatal prognosis, with death occurring in most patients within 5 years of diagnosis. Conventional therapy consists of glucocorticoids or immunosuppressive drugs, which are usually ineffective in preventing the progression of fibrosis (1). A number of studies showed that stem cells derived from adult tissues could differentiate into lung epithelial cells and confer a functional benefit (2-7). Therefore, the contribution of adult stem cells to the regeneration of lung tissue was considered an alternative approach to the treatment of certain lung diseases. However, despite these promising results, studies have failed to restore lung epithelium with adult stem cells (8-10).

Classically, the progenitor cells of the pulmonary epithelium are considered to be the alveolar type II cells in the alveoli.

(Received in original form October 18, 2006; accepted in final form July 19, 2007) Supported by grant FIS PI060679 from the Spanish Ministry of Health and by grant N-2005-79 from the Sociedad Española de Neumología y Cirugía Torácica (SEPAR).

Correspondence and requests for reprints should be addressed to Anna SerranoMollar, Ph.D., Department of Experimental Pathology, IIBB-CSIC-IDIBAPS, C/ Rosselló, 161, 7a, 08036 Barcelona, Spain. E-mail: amsbam@iibb.csic.es

Am J Respir Crit Care Med Vol 176. pp 1261-1268, 2007

Originally Published in Press as DOI: 10.1164/rccm.200610-14910C on July 19, 2007

Internet address: www.atsjournals.org

\section{AT A GLANCE COMMENTARY}

Scientific Knowledge on the Subject

Idiopathic pulmonary fibrosis is a fibroproliferative disorder characterized histopathologically by heterogeneous interstitial fibrosis with fibroblastic foci and honeycomb lung. At present no treatment has proven beneficial to survival.

What This Study Adds to the Field

Transplantation of alveolar type II cells is successful in reversing lung fibrosis in the experimental bleomycin model in rats.

Alveolar type II cells are reparative in nature, rapidly proliferating and differentiating into alveolar type I cells after epithelial cell injury. However, idiopathic lung fibrosis is characterized by the fact that both alveolar type II and type I cells die, and are replaced by abundant fibroblasts (11). On the basis of this evidence, loss of alveolar type II cells compromises the reparative mechanism and is thought to play a significant role in the development and progression of pulmonary fibrosis.

Bleomycin (BLM) instillation in rat lungs causes an inflammatory process that results in pulmonary fibrosis with lesions distributed heterogeneously, resembling the natural progression of the human disease, for example, patchy parenchymal inflammation of variable intensity, epithelial cell injury with reactive hyperplasia, basement membrane damage, and interstitial as well as intraalveolar fibrosis. Hence this model has been widely used to study the regulation of lung fibrosis (12).

In the current investigation, we have performed intratracheal transplantation of isolated alveolar type II cell in a rat model of BLM-induced lung fibrosis to evaluate the ability of these cells to repair damaged lung tissue. We observed the reparative effect of alveolar type II cells transplanted at three different times after fibrosis induction, corresponding to the three different phases of the development of BLM-induced lung fibrosis. The first phase, 3 days after BLM instillation, is characterized by apoptosis and necrosis of alveolar epithelial cells, followed by prominent infiltration of lymphocytes (13). The second phase, 7 days after BLM instillation, is associated with the release of various cytokines that promote the development of lung fibrosis (14-16). The third phase, 15 days after BLM instillation, represents the full development of the disease and is characterized by enhanced collagen deposition inside the lung and by expansion of the lung interstitium resulting from the proliferation of fibroblasts and smooth muscle cells (14-16).

We report here that the transplantation of alveolar type II cells is effective in preventing lung fibrosis. 


\section{METHODS}

\section{Animals}

Syngeneic Lewis rats, weighing $170-200 \mathrm{~g}$ at the beginning of the experiment, were used according to European Community (Directive 86/609/EEC) and Spanish guidelines for the treatment of experimental animals.

\section{BLM-induced Lung Fibrosis}

Pulmonary fibrosis was induced in female animals by intratracheal instillation of a sublethal dose of BLM $(2.5 \mathrm{U} / \mathrm{kg})$ dissolved in $400 \mu \mathrm{l}$ of sterile saline under halothane anesthesia (17). Control animals received the same volume of saline. During the experiment, animal body weights were recorded every 2 days.

\section{Purification of Alveolar Type II Cells}

Fresh alveolar type II cells were isolated from healthy male donor animals. The protocol for purification has been described elsewhere (18). Briefly, to isolate alveolar type II cells, the lungs were removed from each animal and lavaged six times, each time with $8 \mathrm{ml}$ of saline. The lungs were digested with $0.25 \%$ trypsin (T8003; Sigma, St. Louis, MO) dissolved in saline $(100 \mathrm{ml})$ and suspended in $0.9 \% \mathrm{NaCl}$ at $37^{\circ} \mathrm{C}$ for 30 minutes. The trypsin was constantly topped up during the 30 minute expansion of the parenchyma.

After digestion, the lungs were chopped into 1- to 2- $\mathrm{mm}^{2}$ cubes, treated with DNase dissolved in saline $(7,500 \mathrm{U} / 100 \mathrm{ml})$, and filtered through nylon meshes ranging in pore size from 150 to $30 \mu \mathrm{m}$. The resulting cell suspension was centrifuged $\left(250 \times \mathrm{g}, 20 \mathrm{~min}\right.$ at $\left.10^{\circ} \mathrm{C}\right)$ through a sterile Percoll gradient and the alveolar type II cell-rich band was removed. A second DNase treatment $(2,000 \mathrm{U} / 100 \mathrm{ml})$ was administered and the cells were recovered as a pellet by centrifugation at $250 \times \mathrm{g}$ for 20 minutes. These cells were resuspended in $5 \mathrm{ml}$ of DCCM-1 (Biological Industries, Kibbutz Beit Haemek, Israel) supplemented with a $2 \%$ (wt/vol) L-glutamine and subjected to differential attachment on a plastic Petri dish. No adherent alveolar type II cells were collected after 2 hours and cells were counted to establish the final yield of freshly purified cells.

Alveolar type II cell viability was assessed with trypan blue (Sigma), showing greater than $95 \%$ viability. Cell yield and purity was assessed with an alkaline phosphatase staining kit (Sigma). Cells were cytocentrifuged (Cytospin 3; Shandon Scientific Ltd, Grupo Taper, Madrid, Spain) and cell smears were allowed to air dry and were stained for 15 minutes at room temperature. Staining was conducted according to the manufacturer's protocol for the use of alkaline phosphatase (18). Cells were evaluated by transmission electron microscopy. The cells were fixed in $2.5 \%$ glutaraldehyde in phosphate buffer $(0.1 \mathrm{~mol} / \mathrm{L}, \mathrm{pH} 7.4)$ for at least 2 hours at $4^{\circ} \mathrm{C}$. The cells were secondarily fixed in $1 \%$ osmium tetroxide and $0.8 \%$ potassium ferrocyanide for 1 hour at $4^{\circ} \mathrm{C}$. After three washes with cold double-distilled water, the samples were dehydrated through an ascending concentration of acetone $(30,50,70,95$, and $100 \%)$, and three changes of $100 \%$ acetone. They were then embedded in Spurr resin and polymerized at $60^{\circ} \mathrm{C}$. The embedded blocks were sectioned with a diamond knife (Diatome, Biel, Switzerland) on a Leica Ultracut UCT (Leica Microsystems, Deerfield, IL). Ultrathin sections were placed on copper grids and stained with uranyl acetate and lead citrate before examination with a JEM 1010 (JEOL Ltd., Tokyo, Japan) equipped with a Gatan/ BioScan digital camera (Gatan, Inc., Pleasanton, CA).

Cell yield, purity, and characterization of freshly isolated alveolar type II cells were established by the presence of intracellular alkaline phosphatase (Figure 1A), and of lamellar bodies and microvilli (Figures $1 \mathrm{~B}$ and $1 \mathrm{C}$ ) at the electron microscope level. More than $86 \%(\mathrm{n}=4)$ of purified rat cells stained positively for alkaline phosphatase.

\section{Experimental Groups}

Animals were randomly distributed into six experimental groups $(\mathrm{n}=8$ for each group):

Control: Saline instillation

Control plus transplantation: Saline instillation followed by alveolar type II cell transplantation 3 days after instillation
$B L M$ : Bleomycin instillation

$B L M$ plus transplantation $(3 d)$ : Bleomycin instillation plus alveolar type II transplantation 3 days after instillation

BLM plus transplantation ( $7 d$ ): Bleomycin instillation plus alveolar type II transplantation 7 days after instillation

$B L M$ plus transplantation $(15 d)$ : Bleomycin instillation plus alveolar type II transplantation 15 days after instillation

\section{Transplantation Procedure}

On Days 3, 7, and 15 after endotracheal BLM administration, female recipient animals were transplanted with alveolar type II cells endotracheally, by the transoral route under halothane anesthesia. Each animal received a dose of $2.5 \times 10^{6}$ cells suspended in $400 \mu \mathrm{l}$ of sterile saline. The control group received the same dose of cells 3 days after saline instillation. Animals were killed 21 days after induction of lung fibrosis.

\section{Real-time Polymerase Chain Reaction}

The engraftment of donor alveolar type II cells was evaluated by examination of the lung genomic DNA of the transplanted animals, using the real-time polymerase chain reaction (PCR) as described (19) and an iCycler iQ5 real-time PCR detection system (Bio-Rad Laboratories, Hercules, CA) using PCR Y chromosome primers $5^{\prime}$-CTG-GCT-CTGCTC-CTA-CCT-ATG-C-3' and 5'ATT-CTC-CTT-TCT-TAC-ACACTA-CAC-ATC-C-3' and an iQ SYBR Green supermix kit (Bio-Rad Laboratories). Standard curves were generated by serially diluting male rat genomic DNA into female rat genomic DNA prepared from liver.

\section{Fluorescence In Situ Hybridization for the Y Chromosome}

Fluorescence in situ hybridization (FISH) assays of formalin-fixed tissue sections were performed to identify the Y chromosomes. Briefly, paraffin sections of $4 \mu \mathrm{m}$ were deparaffinized and incubated in salinesodium citrate ( $4 \times \mathrm{SSC}$ buffer, $\mathrm{pH} 7.0)$ for 30 minutes, and then the slides were incubated in $1 \mathrm{M}$ sodium isothiocyanate at room temperature overnight. Slices were incubated in pepsin for 5 minutes and washed twice in phosphate-buffered saline (PBS) for 5 minutes, washed once with formaldehyde-PBS- $\mathrm{MgCl}_{2}$ for 1.5 minutes and with PBS for 1.5 minutes, then followed by serial ethanol dehydration steps $(1.5 \mathrm{~min}$ each). Sections were denatured at $65^{\circ} \mathrm{C}$ for 2 minutes in preheated $70 \%$ formamide and $2 \times \mathrm{SSC}$ buffer, $\mathrm{pH} 7.0$, and subsequently "quenched" with ice-cold $70 \%$ ethanol for 1.5 minutes. Serial ethanol dehydration was done again. The rat $\mathrm{Y}$ chromosome probe labeled with fluorescein isothiocyanate (Star*FISH; Cambio, Cambridge, UK) was denatured at $65^{\circ} \mathrm{C}$ for 10 minutes and applied to the sections at $45^{\circ} \mathrm{C}$. The sections were coverslipped and sealed with rubber cement for incubation overnight in a hydrated slide box at $42^{\circ} \mathrm{C}$. Coverslips were carefully removed in preheated $2 \times \mathrm{SSC}$ buffer, $\mathrm{pH} 7.0$, at $45^{\circ} \mathrm{C}$. Sections were preheated in $50 \%$ formamide in $2 \times$ SSC buffer for 5 minutes each at $45^{\circ} \mathrm{C}$ and gently washed twice in preheated $0.1 \times$ SSC buffer for 5 minutes each at $45^{\circ} \mathrm{C}$. Sections were observed by confocal microscopy. Confocal images were acquired with a Leica TCS SL laser-scanning confocal spectral microscope (Leica Microsystems Heidelberg GmbH, Mannheim, Germany) with argon and helium-neon lasers attached to a Leica DMIRE2 inverted microscope. Fluorescein isothiocyanate and propidium iodide emission were acquired sequentially with a triple dichroic beam splitter (TD 488/543/633 nm) and emission detection ranges of $500-535$ and $592-700 \mathrm{~nm}$, respectively. All images were obtained with a $\times 63$ oil immersion objective lens (numeric aperture, 1.32) equipped with phase-contrast optics and a confocal pinhole setting of 1 Airy unit. Because of the small size of the cells an electronic zoom $(\times 2)$ was necessary for stronger magnification and better image resolution. Image assembly and treatment were performed with imageprocessing Leica confocal software.

\section{Hydroxyproline Content}

Lung hydroxyproline content was measured as an indicator of collagen deposition by the method outlined by Woessner (20). Samples were homogenized and then hydrolyzed in $6 \mathrm{M} \mathrm{HCl}$ for 18 hours at $110^{\circ} \mathrm{C}$. The hydrolysate was then neutralized with $2.5 \mathrm{M} \mathrm{NaOH}$. Aliquots 
$(2 \mathrm{ml})$ were analyzed for hydroxyproline content after addition of $1 \mathrm{ml}$ of chloramine-T, $1 \mathrm{ml}$ of perchloric acid, and $1 \mathrm{ml}$ of dimethylaminobenzaldehyde. Samples were read for absorbance at $550 \mathrm{~nm}$ in a spectrophotometer. Results are expressed as micrograms of hydroxyproline per lung.

\section{Histology}

For histologic studies, the left lung was perfused via its main bronchus with a fixative solution ( $10 \%$ neutral-buffered formalin) at a pressure of $25 \mathrm{~cm} \mathrm{H}_{2} \mathrm{O}$ and immersed in the fixative for $12 \pm 24$ hours. Lung tissue blocks were then taken and placed in formalin, dehydrated in a graded series of ethanol, embedded in paraffin, cut into $4-\mu \mathrm{m}$-thick serial sections, and stained with hematoxylin-eosin and Masson's trichrome to identify inflammatory cells, connective tissue, and collagen deposition.

\section{Macrophage Transplantation}

An additional experiment involving macrophage transplantation was performed as a control, using an unrelated cell type to determine whether the fibrotic reversal response was specific for alveolar type II cells. Fresh macrophages were isolated from healthy male donor animals by performing bronchoalveolar lavage. Bronchoalveolar lavage fluid was obtained by washing the lungs four times with $10-\mathrm{ml}$ saline aliquots through a tracheal cannula and centrifuged $(300 \times g, 20$ $\min , 4^{\circ} \mathrm{C}$ ). Cell viability was assessed with trypan blue and was greater than $95 \%$. To evaluate the cell yield and purity and to characterize freshly isolated macrophages, Cytospin preparations were stained with Diff-Quik (Diagnostics Grifols SA, Barcelona, Spain) according to the manufacturer's protocol. More than $95 \%(n=4)$ of rat bronchoalveolar cells were macrophages (see Figure 5A).

On Day 15 after bleomycin instillation, female recipient animals were transplanted with macrophages endotracheally, by the transoral route under halothane anesthesia, at a dose of $2.5 \times 10^{6}$ cells per animal. The cells were suspended in $400 \mu \mathrm{l}$ of sterile saline. The control group received the same dose of cells 15 days after saline instillation. Animals were killed 21 days after the induction of lung fibrosis. The animals were randomly distributed into four experimental groups $(\mathrm{n}=$ 8 for each group):

\section{Control: Saline instillation}

Control plus transplanted macrophages: Saline instillation plus macrophage transplantation 15 days after instillation

$B L M$ : Bleomycin instillation

BLM plus transplanted macrophages $(15 d)$ : Bleomycin instillation plus macrophage transplantation 15 days after instillation

\section{Statistical Analysis}

Data are expressed as means (SEM) with 95\% confidence intervals. Statistical analysis was performed by analysis of variance followed by appropriate post hoc tests including the Newman-Keuls test when differences were significant (GraphPad Software, Inc., San Diego, CA). A $P$ value less than 0.05 was considered significant.

\section{RESULTS}

BLM instillation initially causes a marked loss in body weight, which is normally recovered later. After alveolar type II cell transplantation, animals regained weight more quickly than did BLM nontransplanted animals (Figure 2A). This indicated that alveolar type II cell transplantation enhanced the health of the animals.

After lung collection, the macroscopic appearance of BLM nontransplanted lungs was fibrotic. They were large, yellowish and had several scars when compared with saline control lungs (Figure 2B). In contrast, BLM transplanted lungs showed an improved appearance similar to that of the saline control lungs and were devoid of scars (Figure 2B). Figure 2C shows Masson's trichrome-stained lung sections examined by stereomicroscopy to illustrate the heterogeneous topography of the fibrotic lesions (patchy areas of lung fibrosis); BLM nontransplanted lungs showed extensive areas of fibrosis compared with BLM transplanted lungs.

BLM instillation caused an increase in lung weight owing to the inflammatory and fibrotic component (17). Lung weight was significantly decreased in the transplanted groups 7 and 15 days after BLM instillation compared with the BLM nontransplanted group (Figure 2D).

Because alveolar type II cell transplant donors and recipients were sex-mismatched, engraftment levels of male donor-derived cells in the lungs of female transplant recipients were quantified by real-time PCR (19). No male DNA was detected in the control transplant animals. We found $0.18,0.30$, and $0.49 \%$ male DNA in the transplanted groups 3,7 , and 15 days after BLM, respectively (Figure 3A). These data demonstrate that the engraftment of alveolar type II cells is more effective when fibrosis has developed than in its early phases.

In addition, the successful engraftment of male donor cells in the female transplanted animals was assessed by FISH of the Y chromosome. FISH analysis was performed in paraffin-embedded sections of lung tissue, using Y chromosome paint. Y chromosomepositive FISH signals (absent in the female controls and present in the male controls) were observed in lung sections from all the transplanted groups (Figure 3B). In sections of transplanted lungs, these signals were localized mainly in areas of fibrosis.

BLM administration to rats results in an increase in collagen deposition in the lung. Therefore, the amount of hydroxyproline, a modified amino acid specifically found in collagen (20), was assessed to determine how alveolar type II transplantation could alter the clinical course of BLM-induced lung injury. BLM nontransplanted lungs showed a significant increase in the amount of lung hydroxyproline when compared with saline control rats (Figure 4A). In contrast, levels of hydroxyproline were significantly reduced in all the BLM transplanted groups. In the groups transplanted on Days 7 and 15 after BLM instillation, we observed a greater reduction in hydroxyproline levels, which did not significantly differ from levels observed in the saline control groups. These results confirm that transplantation of alveolar type II cells induces a reduction in collagen deposition and, consequently, in the fibrotic response.

To further examine the effect of alveolar type II cell transplantation in BLM-induced pulmonary fibrosis, serial lung sections were stained with hematoxylin-eosin or Masson's trichrome and examined by light microscopy. Lung tissue sections from control rats showed no evidence of inflammation or epithelial damage. As expected, lung tissue sections from rats with BLMinduced fibrosis (nontransplanted) showed marked peribronchiolar and interstitial infiltration with inflammatory cells, extensive cellular thickening of interalveolar septa, interstitial edema, increased interstitial cells with a fibroblastic appearance, excessive collagen deposition, and focal cuboidal metaplasia of alveolar lining cells (Figure 4B, row a). The pattern of lesions was multifocal (i.e., patchy areas of pulmonary fibrosis) and in most cases involved the pleura. Although multifocal parenchymal lesions were still present in lungs transplanted with alveolar type II cells, the organized fibroblast foci were smaller and considerably less frequent than were observed in nontransplanted animals. The reduction in parenchymal lesions was evidenced by large areas of undamaged tissue with normal alveolar architecture (Figure 4B, row b). Compared with the BLM nontransplanted group, BLM transplanted animals showed less edema, less collagen deposition, less septal widening, and fewer clusters of inflammatory cells.

Specificity of the alveolar type II cells to revert the fibrotic process was verified in a group of animals transplanted with macrophages (Figure 5). When macrophages were used for cell 
A

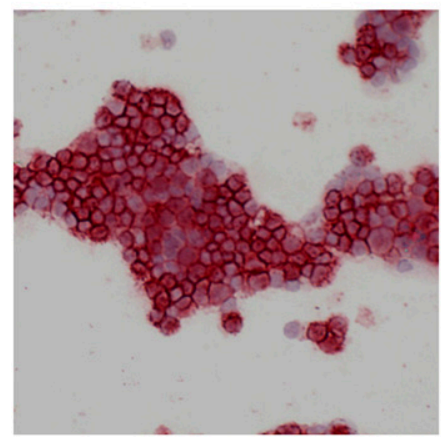

B

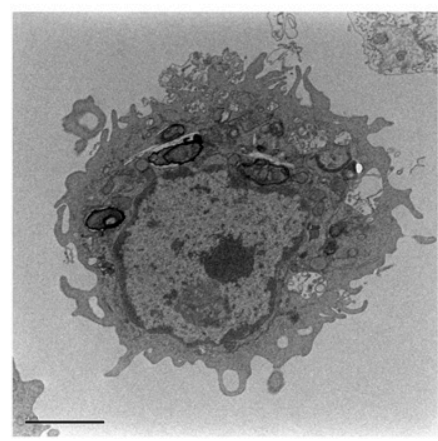

C

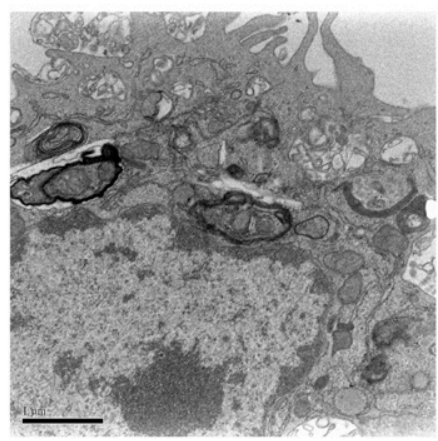

Figure 1. (A) Cytospin preparation showing alkaline phosphatase-positive cells. Original magnification, $\times 200$. (B) Transmission electron micrographs of alveolar type II cells showing lamellar bodies. Bar $=2 \mu \mathrm{m}$. (C) Detail of the lamellar bodies. $B a r=1 \mu \mathrm{m}$. transplantation instead of alveolar type II cells, no beneficial effects were observed. Body weight (Figure 5B), lung weight (Figure 5C), and survival rate showed no significant differences from the BLM nontransplanted group. In addition, hydroxyproline levels remained unmodified (Figure 5D) and the fibrotic areas evaluated by light microscopy after hematoxylin-eosin and Masson's trichrome staining revealed a similar degree of fibrosis compared with that observed in BLM nontransplanted animals.

\section{DISCUSSION}

In this study, we demonstrate for the first time that intratracheal transplantation of alveolar type II cells can halt and reverse lung fibrosis. Alveolar epithelial damage is an important initial event in pulmonary fibrosis. Epithelial cell damage and cell death induce the formation of gaps in the epithelial basement membranes. Migration of fibroblasts through these gaps into the alveolar space leads to intraalveolar fibrosis. Normal repair of the epithelial layer occurs through the proliferation of alveolar type II cells and their subsequent differentiation to alveolar type I cells necessary for proper lung function. In lung fibrosis, however, the regenerative process is altered, with the number of alveolar type II cells decreasing markedly in areas of severe inflammation. Extensive injury and cell death follow $(21,22)$. In areas of severe damage, alveolar type I and type II cells die and are replaced by a large number of fibroblasts and smooth muscle cells. The injury, together with the inadequate repair of
A
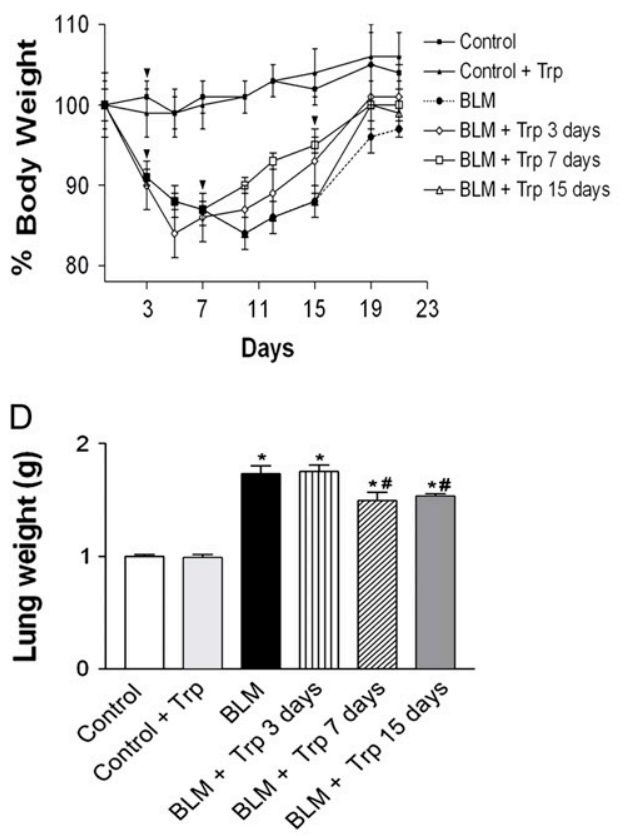

B

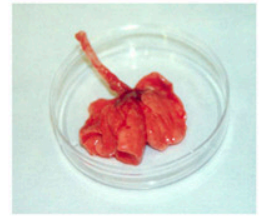

Control

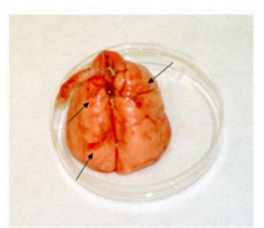

BLM

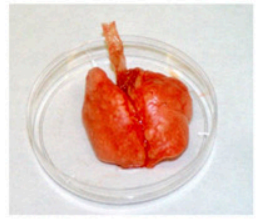

BLM + Trp 7 days

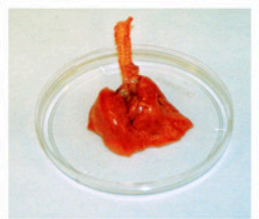

Control + Trp

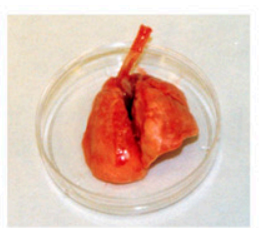

BLM + Trp 3 days

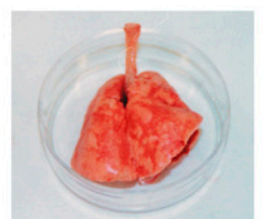

BLM + Trp 15 days

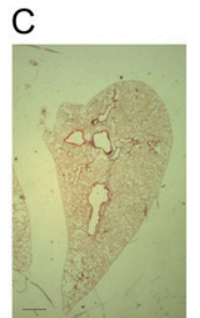

Control

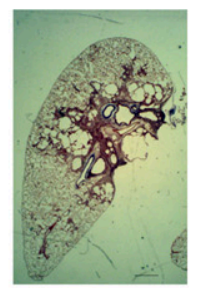

BLM

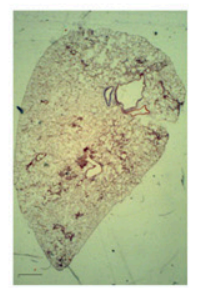

BLM + Trp 3 days
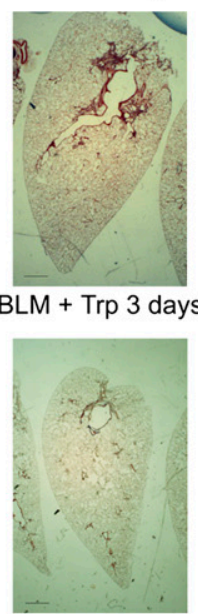

BLM + Trp 7 days BLM + Trp 15 days

Figure 2. (A) Change in animal body weight over time. On Day 0, the animals received bleomycin (BLM). Arrowheads show the days of alveolar type II cell transplantation (Trp) (Days 3, 7, and 15). (B) Representative photographs of whole lungs from all the experimental groups after 21 days of BLM-induced lung fibrosis. Arrows show the scars in the BLM nontransplanted lung. BLM transplanted lungs showed an improved aspect compared with BLM nontransplanted lungs. (C) Representative photographs of lung sections from all the experimental groups. Multifocal parenchymal lesions were smaller in BLM transplanted lungs compared with BLM nontransplanted lungs. Scale bars: $1 \mathrm{~mm}$. (D) Lung weight after 21 days of BLMinduced lung fibrosis in experimental groups. Alveolar type II cells transplanted 7 or 15 days after BLM instillation reduced lung weight. Data represent means \pm SEM of eight animals per group ( ${ }^{*} P<0.05$ vs. control groups, ${ }^{\#} P<0.05$ vs. BLM). 
A

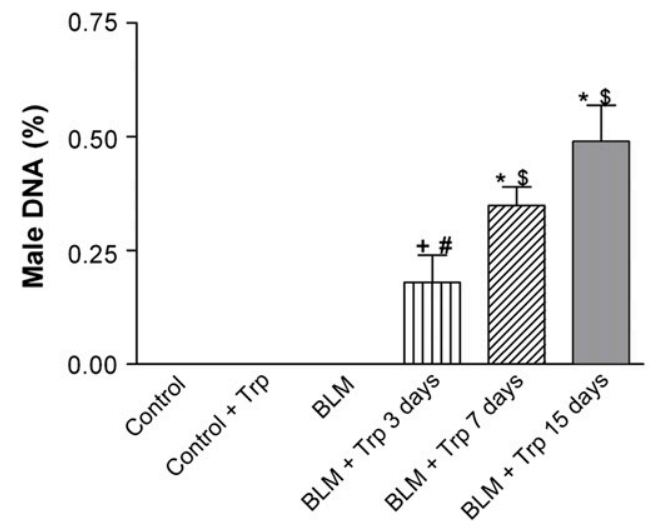

BLM

BLM + Trp 3 days
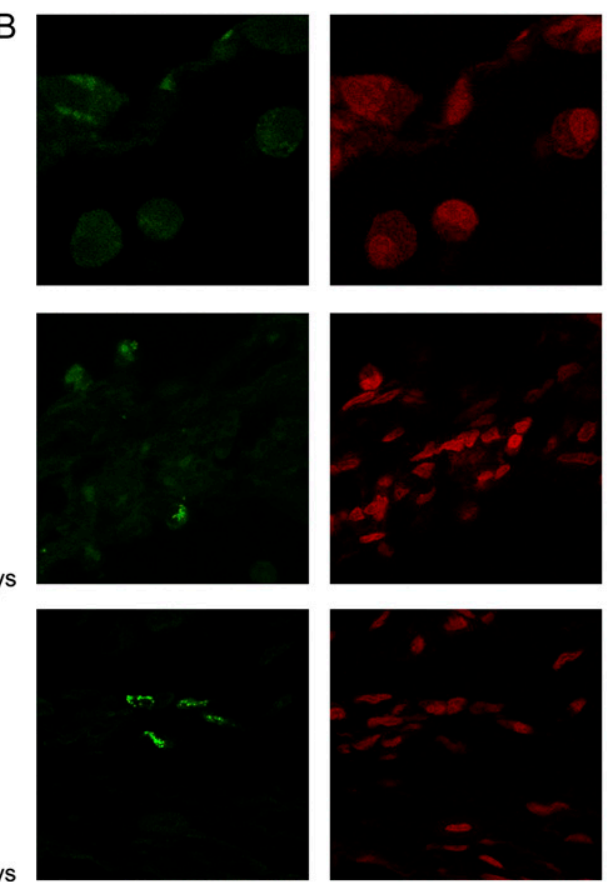

BLM + Trp 7 days

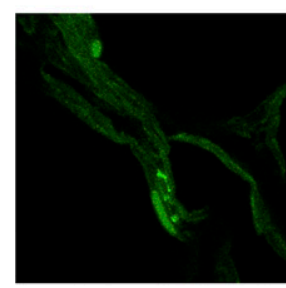

BLM + Trp 15 days
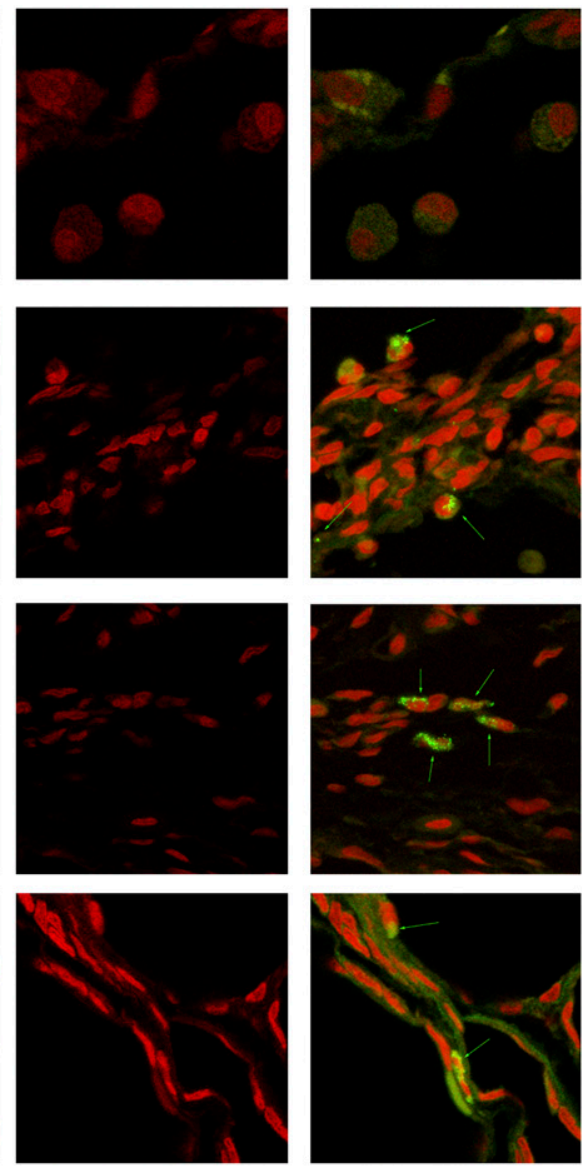

Figure 3. (A) Quantification of alveolar type II cell engraftment in rat lung by real-time polymerase chain reaction (PCR). Histogram of the data obtained in the real-time PCR shows the percentage of male genomic DNA within lung tissue from transplanted rats 21 days after exposure to bleomycin (BLM). Data represent means \pm SEM of eight animals per group $\left({ }^{*} P<0.05\right.$ and $+P<0.001$ vs. control groups, ${ }^{\#} P<0.05$ and $\$ P<$ 0.001 vs. BLM). (B) Detection of donor-derived alveolar type II cells by fluorescence in situ hybridization (FISH) of the Y chromosome. Shown are sections of lung tissue hybridized with fluorescein isothiocyanateconjugated $\mathrm{Y}$ chromosome probe (green dots). Nuclei are counterstained with propidium iodide (red). Arrows indicate representative nuclei that contain the $Y$ chromosome in the transplanted groups. epithelial cells, disturbs normal epithelial-fibroblast interactions and leads to pulmonary fibrosis.

Histologic and biochemical results show that transplantation of alveolar type II cells in rats with BLM-induced lung fibrosis can reverse the fibrogenic response. However, more studies are needed to ascertain the mechanism whereby alveolar type II cells induce recovery in the lung, as several causes could explain this effect. First, the alveolar type II cells could limit the deleterious effects of BLM by replacing already damaged alveolar epithelial type II cells, which are known to be targets of apoptotic signals induced by BLM (22). Alveolar type II cells are known to be the alveolar progenitor cells, which give rise to alveolar type I cells in response to injury (23). Thus, transplantation of alveolar type II cells may restore this pool, leading to an increased number of alveolar cells for the resolution of disrupted alveolar surfaces. In turn, this could promote the repair process. Second, alveolar type II cells have a number of physiological functions, including synthesis, secretion, and turnover of pulmonary surfactant (23). Surfactant maintains low surface tension at the air-liquid interface of the alveoli, which is necessary for proper respiratory function. Transplantation of alveolar type II cells could thus improve the maintenance of normal lung function, promoting normal epithelial repair. Third, alveolar type II cells could protect against BLMinduced fibrosis by altering the lung microenvironment at sites of engraftment, producing a number of cytokine antagonists that disrupt signal pathways leading to fibrosis (23-26). Alveolar type II cells are a rich source of chemokines, including inhibition factors for fibroblast proliferation and secretion of collagen (27). Therefore, secretion of these products from alveolar type II cells could induce degradation of the new collagen deposition, halting the development of lung fibrosis. 


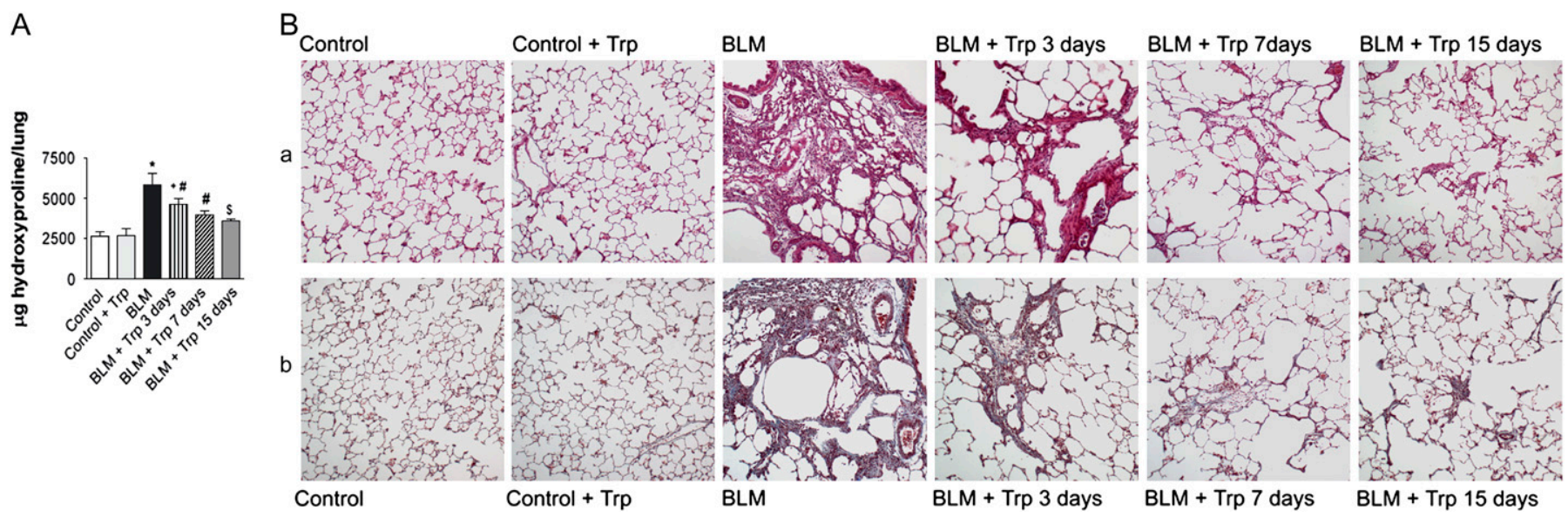

Figure 4. (A) Lung hydroxyproline levels after 21 days of bleomycin (BLM)-induced lung fibrosis. Alveolar type II cell transplantation reduced the lung content of hydroxyproline at all times tested. Data represent means \pm SEM of eight animals per group $\left({ }^{\star} P<0.05\right.$ and ${ }^{+} P<0.001$ vs. control groups, $\# P<0.05$ and $\$ P<0.001$ vs. BLM). (B) Representative photomicrographs of lung histopathology in all the experimental groups after 21 days of BLM-induced lung fibrosis. Row $a$ : Lung sections were stained with hematoxylin-eosin. Alveolar type II cell transplantation ameliorated the inflammatory and pulmonary lesions. Original magnification, $\times 100$. Row $b$ : Masson's trichrome of all the experimental groups. The presence of interstitial collagen (blue staining) was diminished by alveolar type II cells in all the transplantation groups. Original magnification, $\times 100$.
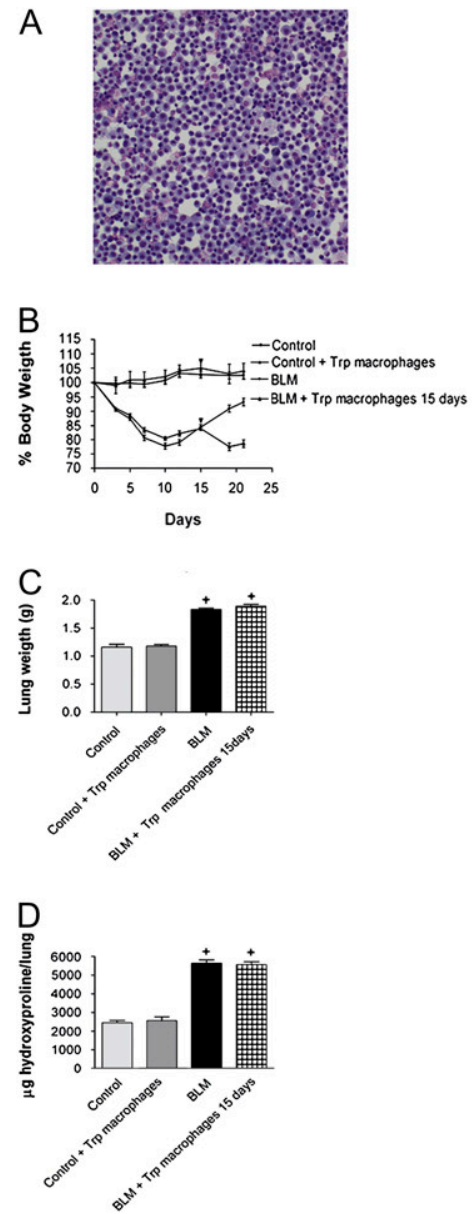
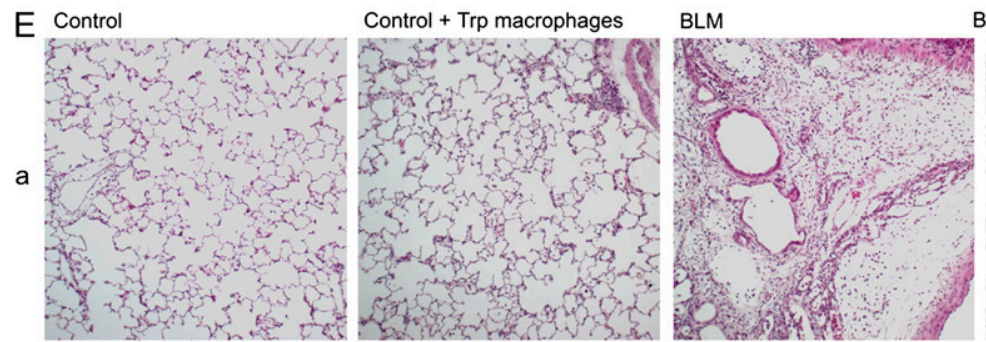

BLM + Trp macrophages 15 days
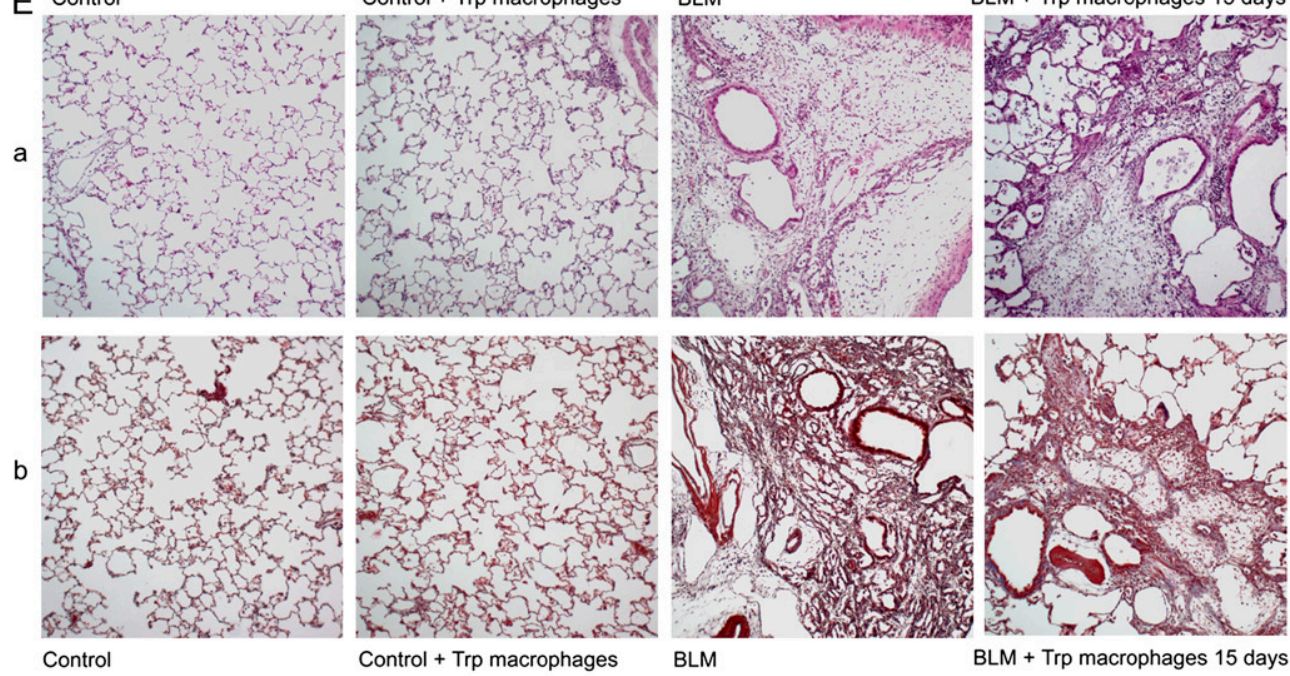

Control + Trp macrophages

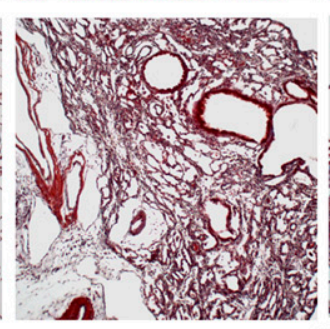

BLM

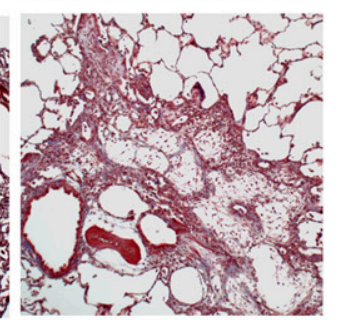

BLM + Trp macrophages 15 days

Figure 5. (A) Cytospin preparation showing alveolar macrophages. Original magnification, $\times 200$. (B) Change in animal body weight over time. On Day 0 the animals received BLM. Arrowheads show the day of macrophage transplantation (Day 15). (C) Lung weight after 21 days of BLM-induced lung fibrosis in the experimental groups. $(D)$ Lung hydroxyproline levels after 21 days of BLM-induced lung fibrosis. Macrophage transplantation did not reduce the lung content of hydroxyproline. Data represent means \pm SEM of eight animals per group $\left({ }^{+} P<0.001\right.$ vs. control groups). ( $E$ ) Representative photomicrographs of lung histopathology in all the macrophage experimental groups after 21 days of BLM-induced lung fibrosis. Row $a$ : Lung sections were stained with hematoxylin and eosin. Macrophage transplantation did not reduce inflammatory and pulmonary lesions. Original magnification, $\times 100$. Row $b$ : Masson's trichrome staining does not show a decrease in the presence of interstitial collagen (blue staining) in the macrophage-transplanted group. Original magnification, $\times 100$. 
The success of the method reported here could be due to the fact that we used adult progenitor cells and the intratracheal route for transplantation. This stands in contrast to earlier reports, in which stem cells derived from adult tissues, such as bone marrow-derived cells and mesenchymal stem cells, were transplanted by the intravenous route (2-6). Previous studies showed that bone marrow-derived cells transplanted intravenously engrafted in different nonhematopoietic tissues and acquired epithelial phenotypes, which suggested a new paradigm for epithelial tissue repair (2). Nevertheless, the initial enthusiasm vanished when subsequent studies either failed to reproduce these initial results (28) or concluded that engraftment events were due to transplanted cells fusing with endogenous somatic cells (28-32). One important caveat relates to the intrinsic structure of the lung, in which each alveolus comprises septae containing capillaries filled with circulating blood cells, which could have been misinterpreted as bone marrow-derived cells engrafted in the lung (33). In this regard, our method using the intratracheal transoral route directly provides the necessary cells for repairing the damaged lung. Because the origin of fibroblasts in pulmonary fibrosis is assumed to be intrapulmonary, the potential extrapulmonary origin of lung fibroblasts in pulmonary fibrosis merits further investigation. Evidence suggests that bone marrow-derived cells migrate to wound-healing sites and serve as sources of fibroblasts and myofibroblasts, which participate in the fibrotic process in response to the signals released during lung injury and fibrosis (1, 2, 8, 33-35). Therefore, it seems reasonable that the increase in these signals could lead to migratory recruitment of transplanted bone marrow-derived cells into the lung and could contribute to the pathogenesis of lung fibrosis. By contrast, administration of alveolar type II cells would provide the necessary cells for the repair process. This notion has been confirmed by transplantation of macrophages, which did not improve the course of lung fibrosis.

One important aspect of this study is the assignment of times for alveolar type II cell transplantation. Transplantation was performed at three different times after BLM-induced lung injury: in the inflammatory phase ( $3 \mathrm{~d}$ after BLM instillation); in the profibrotic phase ( $7 \mathrm{~d}$ after BLM instillation); and in the fibrotic phase (15 d after BLM instillation), when the disease was fully developed. This allowed us to determine the phase in which transplantation could restore the damaged lung. Surprisingly, our data indicate that alveolar type II cell transplantation can halt the fibrogenic process at any of these times. It is important to take into account that at 3 and 7 days after BLM instillation the fibroblasts are not growing yet, and therefore the administration of alveolar type II cells could in this case be preventing the fibrotic process.

For this reason, the most noteworthy result is the significant reduction of fibrosis 15 days after BLM administration, when the lungs are already fibrotic (21). Therefore, our data demonstrate that the transplantation of alveolar type II cells is able to reverse the fibrogenic process. In our opinion, this is a crucial finding given the absence of a useful treatment for this lung disease.

There are multiple therapeutic applications of this work. Because the alveolar type II cells can be obtained by in vitro differentiation of adult stem cells (36) and also by isolation from lung biopsies, it would be possible to perform transplantation in humans. Thus, adult stem cells could constitute an effective and reproducible source of alveolar type II cells, which would allow long-term treatment of lung fibrosis. Moreover, given the propensity of alveolar type II cells to engraft in damaged lung tissue and their ability to restore epithelial damage, these cells could also be an ideal target for gene therapy. Furthermore, because the intratracheal route of administration is commonly employed, this technique would be applicable to clinical practice.

In conclusion, these results show that intratracheal transplantation of alveolar type II cells could become a promising therapy for the future treatment of fibrotic lung diseases.

Conflict of Interest Statement: None of the authors has a financial relationship with a commercial entity that has an interest in the subject of this manuscript.

Acknowledgment: The authors thank Magda Mauri and Nacho Sanchez for technical assistance. The authors also thank Dr. Leticia Fernández, Dr. Esther Roldan, and Angels Serrano for review of the manuscript.

\section{References}

1. American Thoracic Society, European Respiratory Society. International multidisciplinary consensus classification of the idiopathic interstitial pneumonias. Am J Respir Crit Care Med 2002;165:277304.

2. Krause DS, Theise ND, Collector MI, Henegariu O, Hwang S, Gardner R, Neutzel S, Sharkis SJ. Multi-organ, multi-lineage engraftment by a single bone marrow-derived stem cell. Cell 2001;105:369-377.

3. Theise ND, Henegariu O, Grove J, Jagirdar J, Kao PN, Crawford JM, Badve S, Saxena R, Krause DS. Radiation pneumonitis in mice: a severe injury model for pneumocyte engraftment from bone marrow. Exp Hematol 2002;30:1333-1338.

4. Kotton DN, Ma BY, Cardoso WV, Sanderson EA, Summer RS, Williams MC, Fine A. Bone marrow-derived cells as progenitors of lung alveolar epithelium. Development 2001;128:5181-5188.

5. Grove JE, Lutzko C, Priller J, Henegariu O, Theise ND, Kohn DB, Krause DS. Marrow-derived cells as vehicles for delivery of gene therapy to pulmonary epithelium. Am J Respir Cell Mol Biol 2002;27:645-651.

6. Ortiz LA, Gambelli F, McBride C, Gaupp D, Baddoo M, Kaminski N, Phinney DG. Mesenchymal stem cell engraftment in lung is enhanced in response to bleomycin exposure and ameliorates its fibrotic effects. Proc Natl Acad Sci USA 2003;100:8407-8411.

7. Rojas M, Xu J, Woods CR, Mora AL, Spears W, Roman J, Brigham KL. Bone marrow-derived mesenchymal stem cells in repair of the injured lung. Am J Respir Cell Mol Biol 2005;33:145-152.

8. Hashimoto N, Jin H, Liu T, Chensue SW, Phan SH. Bone marrowderived progenitor cells in pulmonary fibrosis. I Clin Invest 2004; 113:243-252.

9. Kotton DN, Fabian AJ, Mulligan RC. Failure of bone marrow to reconstitute lung epithelium. Am J Respir Cell Mol Biol 2005;33: 328-334.

10. Chang JC, Summer R, Sun X, Fitzsimmons K, Fine A. Evidence that bone marrow cells do not contribute to the alveolar epithelium. Am J Respir Cell Mol Biol 2005;33:335-342.

11. Bishop AE. Pulmonary epithelial stem cells. Cell Prolif 2004;37:89-96.

12. Chua F, Gauldie J, Laurent GJ. Pulmonary fibrosis: searching for model answers. Am J Respir Cell Mol Biol 2005;33:9-13.

13. Harrison JH Jr, Hoyt DG, Lazo JS. Acute pulmonary toxicity of bleomycin: DNA scission and matrix protein mRNA levels in bleomycin-sensitive and resistant strains of mice. Mol Pharmacol 1989;36:231-238.

14. Ortiz LA, Moroz K, Liu JY, Hoyle GW, Hammond T, Hamilton RF, Holian A, Banks W, Brody AR, Friedman M. Alveolar macrophage apoptosis and TNF- $\alpha$, but not p53, expression correlate with murine response to bleomycin. Am J Physiol 1998;275:L1208-L1218.

15. Phan SH, Kunkel SL. Lung cytokine production in bleomycin-induced pulmonary fibrosis. Exp Lung Res 1992;18:29-43.

16. Phan SH, Thrall RS, Ward PA. Bleomycin-induced pulmonary fibrosis in rats: biochemical demonstration of increased rate of collagen synthesis. Am Rev Respir Dis 1980;121:501-506.

17. Serrano-Mollar A, Closa D, Prats N, Blesa S, Martinez-Losa M, Cortijo J, Estrela JM, Morcillo EJ, Bulbena O. In vivo antioxidant treatment protects against bleomycin-induced lung damage in rats. $\mathrm{Br} J$ Pharmacol 2003;138:1037-1048.

18. Richards RJ, Davies N, Atkins J, Oreffo VI. Isolation, biochemical characterisation, and culture of lung type II cells of the rat. Lung 1987;165:143-158.

19. McBride C, Gaupp D, Phinney DG. Quantifying levels of transplanted murine and human mesenchymal stem cells in vivo by real-time PCR. Cytotherapy 2003;5:7-18.

20. Woessner JF Jr. The determination of hydroxyproline in tissue and protein samples containing small proportions of this imino acid. Arch Biochem Biophys 1961;93:440-447. 
21. Katzenstein AA, Myers JL. Idiopathic pulmonary fibrosis: clinical relevance of pathologic classification [State of the Art]. Am J Respir Crit Care Med 1998;157:1301-1315.

22. Adamson IY, Bowden DH. Bleomycin-induced injury and metaplasia of alveolar type 2 cells. Am J Pathol 1979;96:531-544.

23. Fehrenbach H. Alveolar epithelial type II cell: defender of the alveolus revisited. Respir Res 2001;2:33-46.

24. Magdaleno SM, Barrish J, Finegold MJ, DeMayo FJ. Investigating stem cells in the lung. Adv Pediatr 1998;45:363-396.

25. Piguet PF, Vesin C. Treatment by human recombinant soluble TNF receptor of pulmonary fibrosis induced by bleomycin or silica in mice. Eur Respir J 1994;7:515-518.

26. Ortiz LA, Lasky J, Hamilton RF Jr, Holian A, Hoyle GW, Banks W, Peschon JJ, Brody AR, Lungarella G, Friedman M. Expression of TNF and the necessity of TNF receptors in bleomycin-induced lung injury in mice. Exp Lung Res 1998;24:721-743.

27. Witherden IR, Vanden Bon EJ, Goldstraw P, Ratcliffe C, Pastorino U, Tetley TD. Primary human alveolar type II epithelial cell chemokine release: effects of cigarette smoke and neutrophil elastase. Am J Respir Cell Mol Biol 2004;30:500-509.

28. Wagers AJ, Sherwood RI, Christensen JL, Weissman IL. Little evidence for developmental plasticity of adult hematopoietic stem cells. Science 2002;297:2256-2259.

29. Alvarez-Dolado M, Pardal R, Garcia-Verdugo JM, Fike JR, Lee HO, Pfeffer K, Lois C, Morrison SJ, Alvarez-Buylla A. Fusion of bone- marrow-derived cells with Purkinje neurons, cardiomyocytes and hepatocytes. Nature 2003;425:968-973.

30. Terada N, Hamazaki T, Oka M, Hoki M, Mastalerz DM, Nakano Y, Meyer EM, Morel L, Petersen BE, Scott E. Bone marrow cells adopt the phenotype of other cells by spontaneous cell fusion. Nature 2002;416:542-545.

31. Murry CE, Soonpaa MH, Reinecke H, Nakajima H, Nakajima HO, Rubart M, Pasumarthi KB, Virag JI, Bartelmez SH, Poppa V, et al. Haematopoietic stem cells do not transdifferentiate into cardiac myocytes in myocardial infarcts. Nature 2004;428:664-668.

32. Balsam LB, Wagers AJ, Christensen JL, Kofidis T, Weissman IL, Robbins RC. Haematopoietic stem cells adopt mature haematopoietic fates in ischaemic myocardium. Nature 2004;428:668-673.

33. Theise ND, Krause DS, Sharkis S. Comment on "Little evidence for developmental plasticity of adult hematopoietic stem cells." Science 2003;299:1317.

34. Blau HM, Brazelton TR, Weimann JM. The evolving concept of a stem cell: entity or function? Cell 2001;105:829-841.

35. Abe R, Donnelly SC, Peng T, Bucala R, Metz CN. Peripheral blood fibrocytes: differentiation pathway and migration to wound sites. J Immunol 2001;166:7556-7562.

36. Van Vranken BE, Romanska HM, Polak JM, Rippon HJ, Shannon JM, Bishop AE. Coculture of embryonic stem cells with pulmonary mesenchyme: a microenvironment that promotes differentiation of pulmonary epithelium. Tissue Eng 2005;11:1177-1187. 